\title{
"Turning a Blind Eye to Ratoon Stunting Disease of Sugarcane in Australia" May Be Putting It Too Strongly Without A Lot More Evidence
}

\author{
J. W. Hoy, ${ }^{\dagger}$ \\ Department of Plant Pathology and Crop Physiology, Louisiana State University Agricultural Center, Baton Rouge, LA 70803
}

A recent Feature article, "Turning a Blind Eye to Ratoon Stunting Disease of Sugarcane in Australia" by Dr. Anthony J. Young, claims "The potential RSD plays a significant, industry-wide role in reduced yields and crop deterioration in Australia has been widely overlooked." As a result, "the industry has exhibited what amounts to an ideological attitude, repeatedly claiming the disease is economically managed." These statements are the basis for the provocative title of the article. Dr. Young presents very elaborately constructed arguments that conclude RSD is likely to be more widespread than realized, is causing significant yield losses and loss of varieties, and is the unrecognized cause of multiple yield decline problems. His overall contention is that the RSD situation has been mismanaged to the detriment of the industry. Allegations such as these should be supported by well documented, direct evidence. Are they supported by solid evidence? A careful, objective evaluation of the contents of this article would conclude the answer is no. Most of Dr. Young's arguments make extensive use of extrapolation and inference of results from the literature, some of which could be interpreted differently. Should unsubstantiated allegations such as these be published in a Plant Disease Feature article? Again, I think the answer should be no.

I am the senior author of the study cited to provide direct comparative evidence that the evaporative-binding enzyme-immunoassay (EB-EIA) does not detect RSD as well as some other methods. Dr. Young makes a convincing case that there is reason for concern, and there is a valid argument to move away from reliance on EBEIA. He provides evidence from his own research that there is a PCR-based method, leaf sheath biopsy-qPCR (LSB-qPCR), which is more sensitive and accurate for RSD detection. This is potentially a very significant contribution.

There are apparently no comprehensive field survey data regarding the incidence of RSD in the Australian sugarcane industry. Dr. Young expresses a legitimate concern that efforts to determine the situation may have been inadequate, yet he has not used the promising LSB-qPCR method that he developed to obtain the needed information. Accurately assessing a cryptic disease like RSD in such a large industry is a huge challenge. Nevertheless, the yield limiting potential of RSD would justify a comprehensive effort to determine whether the current farm-based healthy seed cane approach is effectively managing the disease. Using his pooled sample method as a starting point to evaluate incidence in the industry at the field level, then using single-leaf qPCR to evaluate within-field incidence might be feasible. Whether qPCR would be practical as a tool for routine, industry-wide management of RSD is another question. At this stage, it would be appropriate to pursue funding (he should be able to make an excellent case for it) and do the needed survey to evaluate the situation and get solid evidence - before publishing a Feature article in Plant Disease.

Dr. Young provides one example, in which he was directly involved, where higher yields were obtained in the Harwood region following an effort to enhance RSD management by employing the LSB-PCR diagnostic method, expanding increase plots, and improving distribution of healthy seed cane. He proposes that high yields in two following seasons are "evidence for a significant underlying RSD problem." Yet he acknowledges that this district had a higher RSD incidence historically (and therefore might be expected to show improvement in response to improved management), and then he does not provide any before or after disease incidence results to support his conclusion that the higher yields were the result of a reduction in RSD. Without these data, his conclusion cannot be verified.

Dr. Young eventually suggests RSD is the overlooked explanation for all phenomena associated with yield loss in sugarcane both old and new - without providing any scientific evidence. Instead, he constructs elaborate conjectures based on multilayered suppositions. Why is Plant Disease publishing an "it may be argued that," "could it be," "it may be," "may have contributed," "it is entirely possible," "it is therefore not unlikely" article? Proof is often elusive in research addressing problems with complex etiology, but little or no direct evidence is provided to support any of these conjectures. Dr. Young then makes an elaborate impassioned argument that breeding for resistance will be the solution to the problem. But no breeding program is going to undertake a major, costly selection effort without strong evidence of a problem. The plants being produced through tissue culture in the most recent healthy seed cane scheme might be infected by the pathogen at the beginning of the process - or they might not. This is the case throughout this article. He might be right in his suppositions, or he might be wrong. In the absence of conclusive evidence, it is premature to publish this speculation.

Dr. Young has the opportunity to make a very important contribution to the Australian sugarcane industry regarding the impact and effective management of ratoon stunting disease. He makes a strong case that a comprehensive effort is needed. Therefore, he needs to develop a cooperative effort to go test the fields starting with a focus on the end of the healthy seed cane pipeline (as he suggests). He needs to determine the situation, draw informed conclusions, and make appropriate recommendations. Then, it will be time to publish a Plant Disease Feature article (leaving out the extensive, unsubstantiated speculation concerning additional possible roles of RSD in other complex problems).

${ }^{\dagger}$ Corresponding author: J. W. Hoy; E-mail: jhoy @agcenter.lsu.edu

Accepted for publication 28 October 2018.

() 2019 The American Phytopathological Society 